\title{
Mean pion multiplicities in Ar+Sc collisions
}

\section{Michał Naskręt* for the NA61/SHINE collaboration.}

University of Wroclaw

E-mail: michal.naskreteuwr.edu.pl

\begin{abstract}
NA61/SHINE is a large acceptance fixed target experiment at the CERN SPS which studies final hadronic states in interactions between various particles and nuclei [1]. Preliminary results for mean negatively charged pion multiplicities $\left\langle\pi^{-}\right\rangle$using the $h^{-}$method are presented for central Ar+Sc collisions at 13, 19, 30, 40, 75 and 150A GeV/c beam momentum. The data were taken recently by the NA61/SHINE collaboration. Measured rapidity distributions $\frac{d n}{d y}$ were extrapolated to unmeasured regions to obtain total multiplicities $\left\langle\pi^{-}\right\rangle$. A new scheme to calculate the mean number of wounded nucleons $\langle W\rangle$ utilizing the EPOS MC model is described. Using also data from other experiments, a comparison of the ratio $\frac{\langle\pi\rangle}{\langle W\rangle}$ for different collisions and beam momenta is discussed.
\end{abstract}

Critical Point and Onset of Deconfinement - CPOD2017

7-11 August, 2017

The Wang Center, Stony Brook University, Stony Brook, NY

${ }^{*}$ Speaker. 


\section{Introduction}

NA61/SHINE is a large acceptance fixed target experiment which studies final hadronic states in interactions between various particles and nuclei [1]. The study of central $\mathrm{Ar}+\mathrm{Sc}$ collision measurements is an important part of the physics programme of the NA61/SHINE experiment. Results from the analysis of the data will help to understand the physics of the onset of deconfinement and contribute to the search for the critical point of strongly interacting matter. Among the many different hadrons produced in high energy collisions, pions are the lightest and the most numerous. Thus, data on pion production properties is crucial for constraining the basic properties of models of strong interactions.

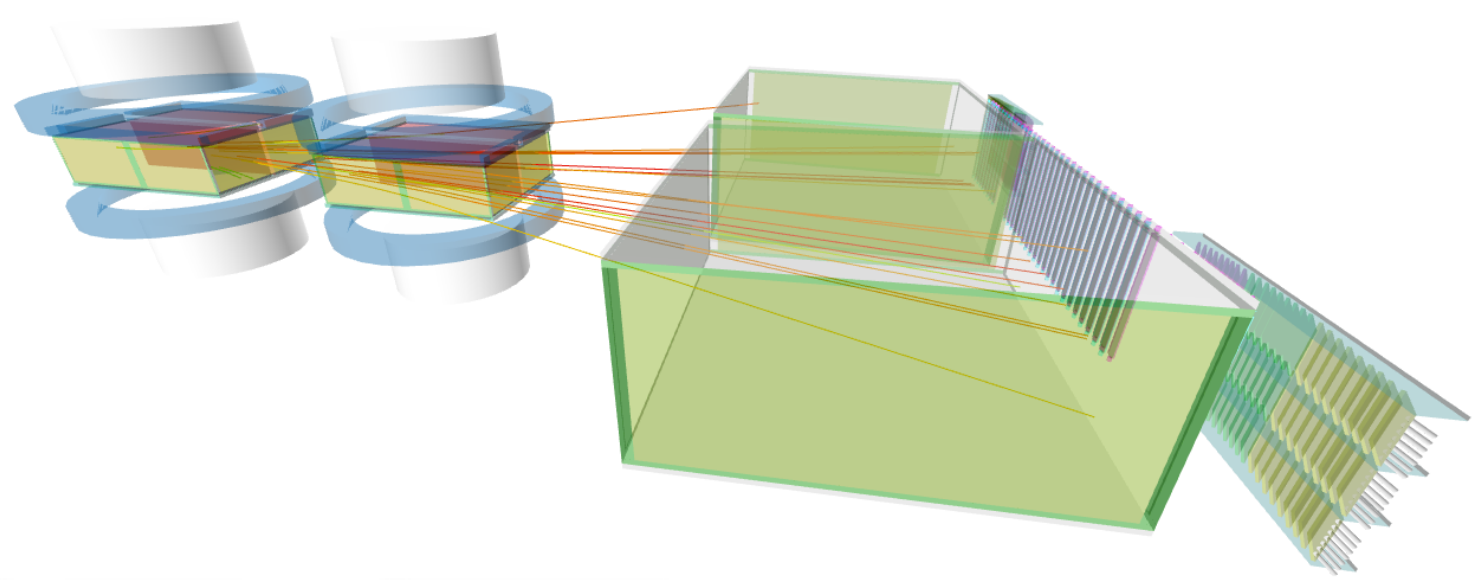

Figure 1: An example of an Ar+Sc collision visualized by the shine3D app.

\section{2. $\pi^{-}$rapidity distributions}

The starting point of the analysis described herein are double differential spectra $\frac{d^{2} n}{d y d p_{\mathrm{T}}}$ of negatively charged hadrons, where $y$ and $p_{\mathrm{T}}$ are rapidity and transverse momentum of particles, respectively. To identify negatively charged pions, the " $\mathrm{h}^{-"}$ method (see details in Ref. [3] and schematic overview in Fig. 2) is used. Central collisions were identified by selecting the 5\% of collisions with the smallest forward going energy as measured by the Projectile Spectator Detector (PSD) [1]. The acceptance of the detector extends to $2 \mathrm{GeV} / \mathrm{c}$ in $p_{T}$ and 3.5 in $y$.

The spectra were obtained from all reconstructed tracks applying a series of quality cuts. In order to correct for trigger and reconstruction inefficiencies, one needs to apply a Monte Carlo correction. To this end, the EPOS MC [4] is used in NA61/SHINE. Large statistics of ion collisions were generated and negatively charged particles were accumulated in bins $n_{\text {gen }}^{i, j}$ in transverse momentum $p_{\mathrm{T}}$ versus rapidity $y$. The simulated events were also processed by the regular reconstruction procedure. Reconstructed negatively charged pions, subjected to the same selection criteria as the real data, were then used to accumulate the distribution $n_{\text {sel }}^{i, j}$. The correction factor $c^{i, j}$ is then calculated as the ratio of the two Monte-Carlo generated spectra $c^{i, j}=n_{\text {gen }}^{i, j} / n_{\text {sel }}^{i, j}$. The final experimental spectra are obtained as $n^{i, j}=n_{\text {data }}^{i, j} c^{i, j} / N_{\text {Event }}$, where $N_{\text {Event }}$ is the total number of events in experimental data. 
Raw data
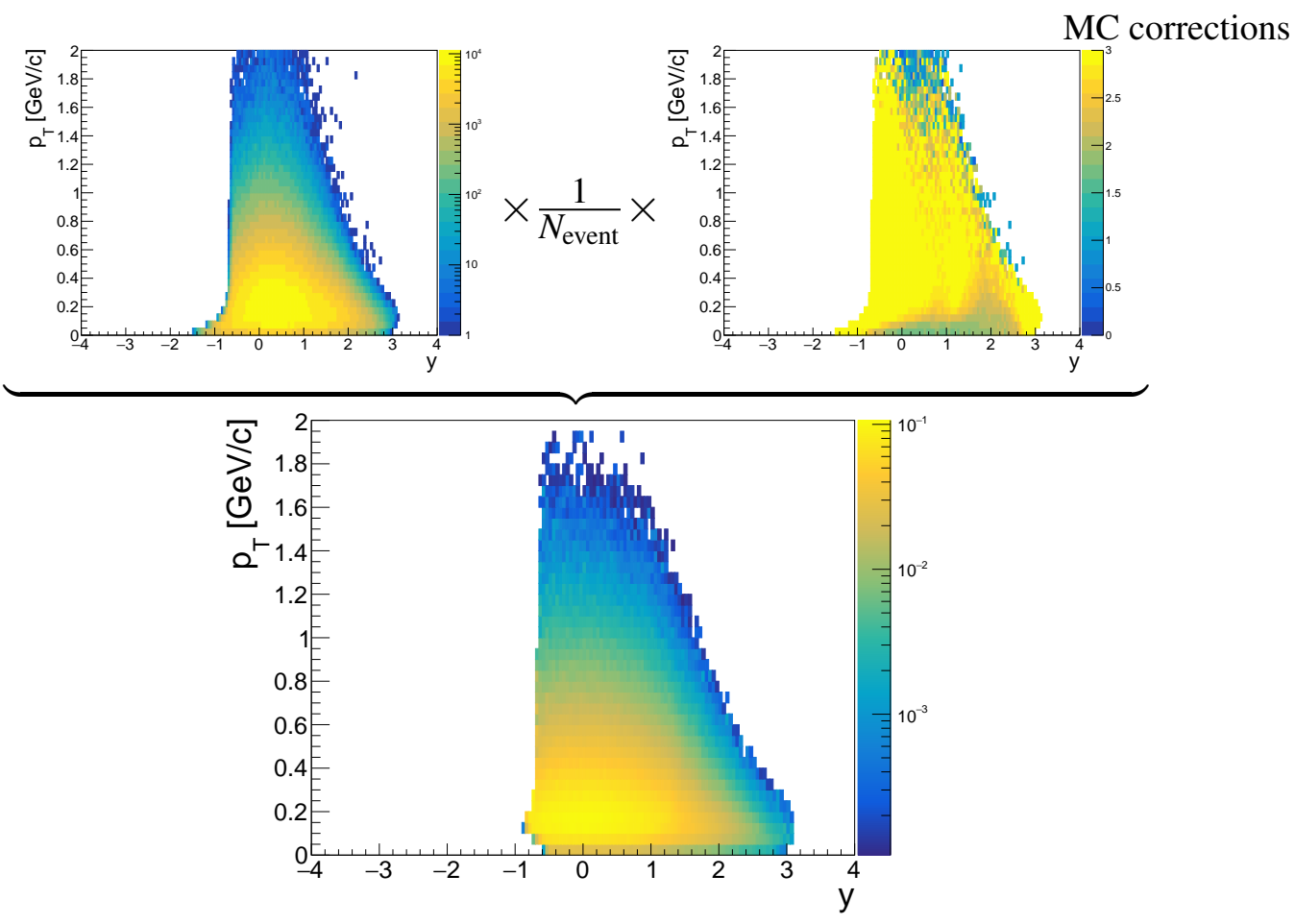

Corrected $\pi^{-}$spectrum

Figure 2: Schematic overview of the $h^{-}$method. Example for central Ar+Sc collisions at 19A GeV/c.

In order to estimate the mean $\pi^{-}$multiplicity in the full acceptance, one needs to extrapolate the experimental data to unmeasured regions. The extrapolation process consisted of two steps: extrapolation in transverse momentum $p_{\mathrm{T}}$ for each bin of rapidity $y$ and extrapolation of $d n / d y$ in rapidity. For the latter a sum of two Gaussian functions was fitted, $g(y)=g_{\mathrm{T}}(y)+g_{\mathrm{P}}(y)$, where

$$
\begin{aligned}
& g_{\mathrm{T}}(y)=\frac{A_{0} A_{r e l}}{\sigma_{0} \sqrt{2 \pi}} \exp \left(-\frac{\left(y-y_{0}\right)^{2}}{2 \sigma_{0}^{2}}\right) \\
& g_{\mathrm{P}}(y)=\frac{A_{0}}{\sigma_{0} \sqrt{2 \pi}} \exp \left(-\frac{\left(y+y_{0}\right)^{2}}{2 \sigma_{0}^{2}}\right)
\end{aligned}
$$

In order to calculate the mean negatively charged pion multiplicity $\left\langle\pi^{-}\right\rangle$, the following formula was utilized:

$$
\left\langle\pi^{-}\right\rangle=\int_{-4}^{y_{\min }} g(y) d y+\sum_{y_{\min }}^{y_{\max }} d y\left(\frac{d n}{d y}\right)_{\text {extrapolated in } p_{\mathrm{T}}}+\int_{y_{\max }}^{4} g(y) d y
$$

Thus the final result is the sum over measured values of $d n / d y$ in the acceptance region and the integral over the Gaussian fits outside. Statistical uncertainties were calculated and systematic uncertainties were assumed to be 5\% based on the previous NA61 analysis of $p+p$ collisions [2]. 


\begin{tabular}{cl|cc} 
& $p_{\text {lab }}[A \mathrm{GeV} / \mathrm{c}]$ & $\left\langle\pi^{-}\right\rangle$ & $\langle W\rangle$ \\
\hline \multirow{4}{*}{$\mathrm{Ar}+\mathrm{Sc}$} & 13 & $38.46 \pm 1.92$ & $66.63 \pm 0.50$ \\
& 19 & $48.03 \pm 2.40$ & $66.68 \pm 1.02$ \\
& 30 & $59.72 \pm 2.98$ & $66.72 \pm 0.50$ \\
& 40 & $66.28 \pm 3.31$ & $66.64 \pm 0.57$ \\
& 75 & $86.12 \pm 4.30$ & $66.66 \pm 0.52$ \\
& 150 & $108.92 \pm 5.44$ & $66.88 \pm 0.50$
\end{tabular}

Table 1: Preliminary results on $\left\langle\pi^{-}\right\rangle$and $\langle W\rangle$ for the $5 \%$ most central Ar+Sc collisions.

\section{Obtaining the mean number of wounded nucleons}

The number of wounded nucleons can not be measured experimentally in NA61/SHINE. It has to be calculated with the help of Monte Carlo models. Three models were used: Glissando 2.73 [5] based on the Glauber model, Hijing 1.0 [6] and EPOS 1.99 (version CRMC 1.5.3) [4] based on a parton ladder model. Glissando provides values that are consistent with previous measurements and the wounded nucleon model [7]. EPOS, on the other hand, allows for more detailed centrality analysis and event selection. It is possible to extract Glauber-based $\langle W\rangle$ values in EPOS which are in good agreement with results from Glissando and Hijing as shown in Fig. 3 with combined statistical and systematic uncertainties. Therefore, Glauber-based EPOS values of $\langle W\rangle$ are used in later considerations.

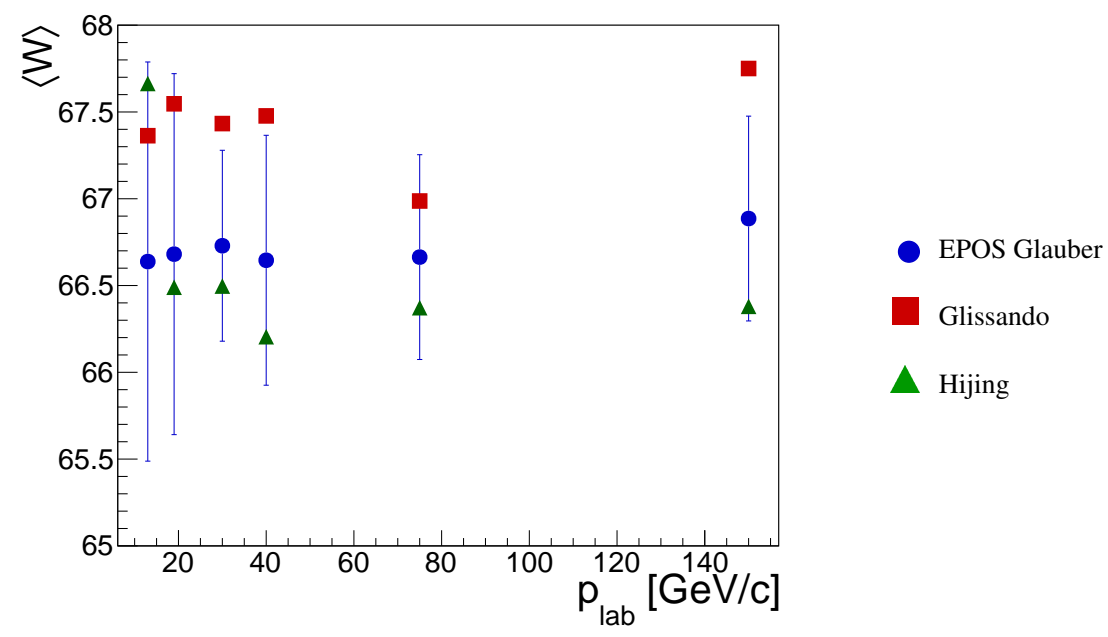

Figure 3: Average number of wounded nucleons $\langle W\rangle$ calculated by Glissando, Hijing and EPOS "a la Glauber".

\section{Results}

Preliminary $\pi^{-}$rapidity spectra for central Ar+Sc collisions are presented in Fig. 4

Preliminary results on $\left\langle\pi^{-}\right\rangle$and $\langle W\rangle$ were calculated according to the procedures described in sections 2 and 3. These are presented in Table 1. 




Figure 4: Preliminary $\pi^{-}$rapidity spectra for the $5 \%$ most central $\mathrm{Ar}+\mathrm{Sc}$ collisions.

In order to compare results obtained for different systems, an isospin correction should be taken into account. To this end the following phenomenological formulas are used:

$$
\begin{gathered}
\left\langle\pi^{-}\right\rangle_{\mathrm{N}+\mathrm{N}}=\left\langle\pi^{-}\right\rangle_{\mathrm{p}+\mathrm{p}}+\frac{1}{3} \\
\left\langle\pi^{-}\right\rangle_{\mathrm{Au}+\mathrm{Au}}^{\mathrm{I}}=\left(\left\langle\pi^{-}\right\rangle_{\mathrm{Au}+\mathrm{Au}}+\left\langle\pi^{+}\right\rangle_{\mathrm{Au}+\mathrm{Au}}\right) / 2
\end{gathered}
$$

The correction is based on the compilation of the world data presented in [8] and the model presented therein and is only applied to measurements where its effect is significant. Where needed, the data is also corrected for slight differences in beam momentum. Applying these corrections one can plot the ratio $\left\langle\pi^{-}\right\rangle /\langle W\rangle$ for different systems for $30 A$ and $150 A \mathrm{GeV} / \mathrm{c}$, see Fig. 5. Comparison of the preliminary $\mathrm{Ar}+\mathrm{Sc}$ results with those from other systems suggest a monotonic increase with $\langle W\rangle$ at $150 \mathrm{~A} \mathrm{GeV/c}$ and a constant value at $30 \mathrm{~A} \mathrm{GeV/c}$.

The Fermi statistical model predicts a linear increase of $\langle\pi\rangle /\langle W\rangle$ with the Fermi energy measure $F=\left[\left(\sqrt{s_{\mathrm{NN}}}-2 m_{\mathrm{N}}\right)^{3} / \sqrt{s_{\mathrm{NN}}}\right]^{1 / 4}$. An increase of the slope of $\langle\pi\rangle /\langle W\rangle$ versus $F$, the "kink", is predicted by the SMES [9] model at the onset of deconfinement due to the larger number of effective degrees of freedom in the deconfined phase than in the hadron resonance gas (HRG).

For central $\mathrm{Ar}+\mathrm{Sc}$ and $\mathrm{Be}+\mathrm{Be}$ collisions as well as $\mathrm{p}+\mathrm{p}$ interactions NA61 could only obtain the value of total multiplicity $\left\langle\pi^{-}\right\rangle$. Multiplicities $\left\langle\pi^{+}\right\rangle$and $\left\langle\pi^{0}\right\rangle$ are approximated by multiplying the previously isospin asymmetry corrected $\pi^{-}$multiplicities by a factor 3 : $\langle\pi\rangle=3\left\langle\pi^{-}\right\rangle$. This approach is necessitated by the fact that the NA61/SHINE acceptance is the largest for $\pi^{-}$. The energy dependence of the ratio $\langle\pi\rangle /\langle W\rangle$ is presented in Fig. 6. The Ar+Sc results follow those from $\mathrm{p}+\mathrm{p}$ for low energies whereas for high energies they follow the measurements from $\mathrm{Pb}+\mathrm{Pb}$. No simple systematics are observed at low SPS energies. This might be caused by different physics processes and/or systematic bias in the estimate of $\langle W\rangle$. Full simulation of the fragmentation process and PSD response is needed in order to reduce the latter uncertainty.

\section{Conclusions}

Preliminary results on $\pi^{-}$rapidity spectra and multiplicities in central Ar+Sc collisions at the 




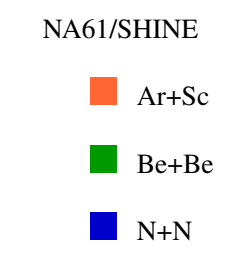

NA49

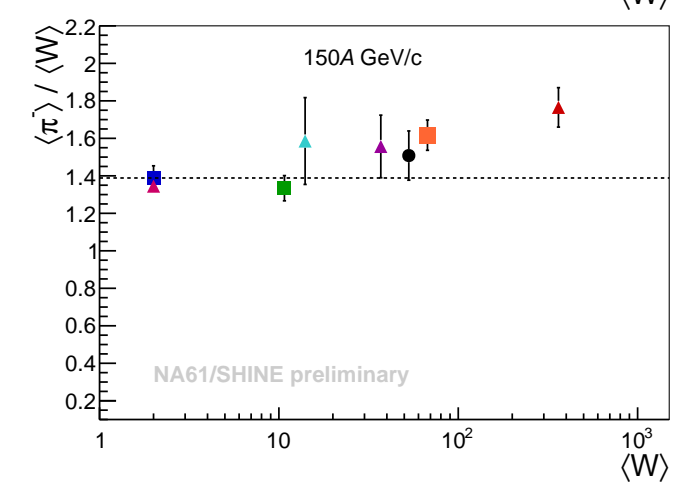

$\triangle \mathrm{Pb}+\mathrm{Pb}$

$\triangle \mathrm{Si}+\mathrm{Si}$

$\triangle \mathrm{C}+\mathrm{C}$

A $N+\mathrm{N}$

NA35

$\mathrm{S}+\mathrm{S}$

Figure 5: Measurements of the ratio $\left\langle\pi^{-}\right\rangle /\langle W\rangle$ in nucleon-nucleon and central nucleus-nucleus collisions.

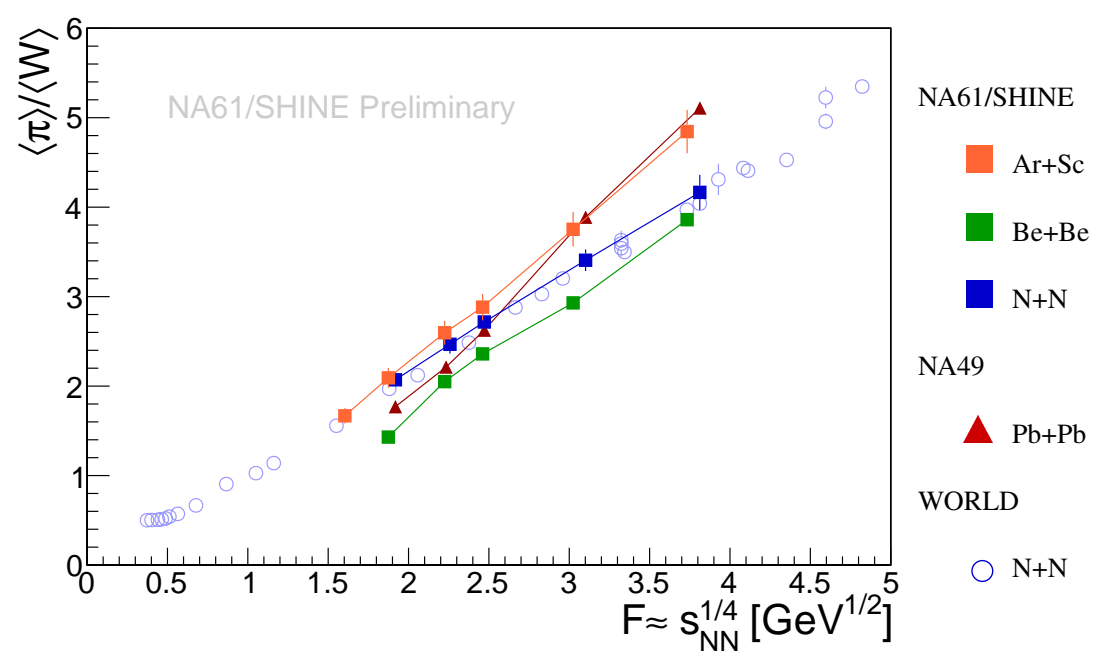

Figure 6: Energy dependence of the ratio $\langle\pi\rangle /\langle W\rangle$ - the kink plot.

CERN SPS are presented. Total multiplicities as function of collision energy and ratios with the average number of wounded nucleons were obtained. These new results are compared to previous measurements on nucleon-nucleon and nucleus-nucleus interactions. Results from Ar+Sc follow those obtained in $\mathrm{Pb}+\mathrm{Pb}$ collisions at higher SPS energies whereas at low energies they follow the measurements in $\mathrm{p}+\mathrm{p}$ reactions.

This work was partially supported by the National Science Centre, Poland (grant 2015/18/M/ST2/00125). 


\section{References}

[1] NA61 collaboration, N. Abgrall et al., NA61/SHINE facility at the CERN SPS: beams and detector system, JINST 9 (2014) P06005, [1401.4699].

[2] NA61/SHINE collaboration, N. Abgrall et al., Measurement of negatively charged pion spectra in inelastic $p+p$ interactions at $p_{l a b}=20,31,40,80$ and $158 \mathrm{GeV} / \mathrm{c}$, Eur. Phys. J. C74 (2014) 2794, [1310.2417].

[3] A. Aduszkiewicz, Energy dependence of negatively charged pion production in proton-proton interactions at the CERN SPS, Ph.D. thesis, University of Warsaw, 8, 2015.

[4] K. Werner, F.-M. Liu and T. Pierog, Parton ladder splitting and the rapidity dependence of transverse momentum spectra in deuteron-gold collisions at RHIC, Phys. Rev. C74 (2006) 044902, [hep-ph/0506232].

[5] M. Rybczyński et al., GLISSANDO 2 : GLauber Initial-State Simulation AND mOre..., ver. 2 , Comput. Phys. Commun. 185 (2014) 1759-1772, [1310.5475].

[6] M. Gyulassy and X.-N. Wang, HIJING 1.0: A Monte Carlo program for parton and particle production in high-energy hadronic and nuclear collisions, Comput. Phys. Commun. 83 (1994) 307, [nucl-th/9502021].

[7] A. Białas, M. Bleszyński and W. Czyż, Multiplicity distributions in nucleus-nucleus collisions at high energies, Nuclear Physics B 111 (1976) 461 - 476.

[8] A. I. Golokhvastov, Koba-nielsen-olesen scaling, Physics of Atomic Nuclei 64 (Jan, 2001) 84-97.

[9] M. Gazdzicki and M. I. Gorenstein, On the early stage of nucleus-nucleus collisions, Acta Phys. Polon. B30 (1999) 2705, [hep-ph/9803462]. 